\title{
Computational Study of the CC3 Impeller and Vaneless Diffuser Experiment
}

\author{
Sameer Kulkarni* \\ NASA Glenn Research Center, Cleveland, Ohio, 44135, USA \\ Timothy A. Beach ${ }^{\dagger}$ \\ Coyote Hollow Consulting LLC, Walton Hills, Ohio, 44146, USA \\ and \\ Gary J. Skoch ${ }^{\ddagger}$ \\ U. S. Army Research Laboratory, Cleveland, Ohio, 44135, USA
}

\begin{abstract}
Centrifugal compressors are compatible with the low exit corrected flows found in the high pressure compressor of turboshaft engines and may play an increasing role in turbofan engines as engine overall pressure ratios increase. Centrifugal compressor stages are difficult to model accurately with RANS CFD solvers. A computational study of the CC3 centrifugal impeller in its vaneless diffuser configuration was undertaken as part of an effort to understand potential causes of RANS CFD mis-prediction in these types of geometries. Three steady, periodic cases of the impeller and diffuser were modeled using the TURBO Parallel Version 4 code: 1) a k- $\varepsilon$ turbulence model computation on a 6.8 million point grid using wall functions, 2) a $k-\varepsilon$ turbulence model computation on a 14 million point grid integrating to the wall, and 3) a k- $\omega$ turbulence model computation on the 14 million point grid integrating to the wall. It was found that all three cases compared favorably to data from inlet to impeller trailing edge, but the $k-\varepsilon$ and $k-\omega$ computations had disparate results beyond the trailing edge and into the vaneless diffuser. A large region of reversed flow was observed in the $k-\varepsilon$ computations which extended from $70 \%$ to $100 \%$ span at the exit rating plane, whereas the k- $\omega$ computation had reversed flow from $95 \%$ to $100 \%$ span. Compared to experimental data at near-peak-efficiency, the reversed flow region in the $k-\varepsilon$ case resulted in an under-prediction in adiabatic efficiency of 8.3 points, whereas the k- $\omega$ case was 1.2 points lower in efficiency.
\end{abstract}

\section{Nomenclature}

$\begin{array}{ll}\text { CFD } & \text { computational fluid dynamics } \\ \mathrm{CMOTT} & =\text { Center for Modeling of Turbulence and Transition } \\ \mathrm{k} & =\text { turbulence kinetic energy (TKE) } \\ \mathrm{R} & =\text { radius } \\ \mathrm{R}_{\mathrm{TE}} & =\text { radius of impeller trailing edge }(215.5 \mathrm{~mm}) \\ \mathrm{RANS} & =\text { Reynolds-averaged Navier Stokes equations } \\ \mathrm{y}^{+} & =\text {dimensionless wall distance } \\ \varepsilon & =\text { dissipation of turbulence kinetic energy } \\ \omega & =\text { specific turbulence dissipation }\end{array}$

\footnotetext{
* Aerospace Engineer, Turbomachinery \& Heat Transfer Branch, sameer.kulkarni@nasa.gov

${ }^{\dagger}$ Engineer, Turbomachinery \& Heat Transfer Branch

‡ Aerospace Engineer, Turbomachinery \& Heat Transfer Branch
} 


\section{Introduction}

C entrifugal compressors are frequently used in the high pressure compressor of turboshaft engines due to the low exit corrected flow rates in these machines. With the decreasing size of the high pressure compressor in turbofan engines as engine overall pressure increases, exit corrected flow rates may be sufficiently low such that the use of a centrifugal stage at the compressor exit may provide performance benefits over a typical axial compressor stage. ${ }^{1}$ Thus, the ability to accurately simulate the flow within centrifugal stages is becoming increasingly important for compressor designers and researchers. However, Reynolds-averaged NavierStokes (RANS)-based computational fluid dynamics (CFD) results published in the literature tend to mispredict the performance of centrifugal compressors when compared to available data. ${ }^{2-5}$ In general, the computations tend to over-predict total pressure ratio, efficiency, and in the case of vaned diffuser

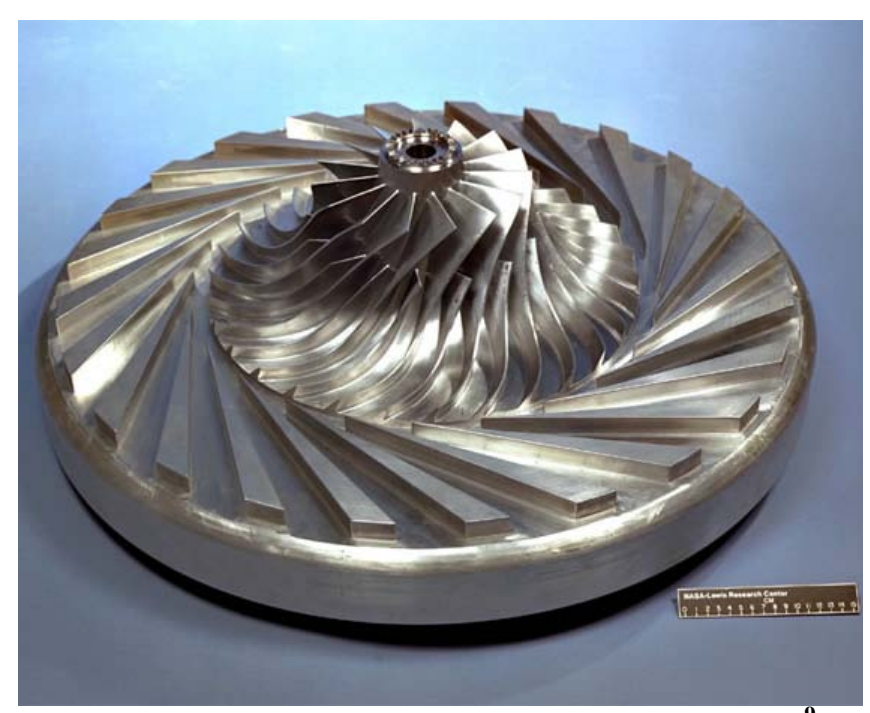

Figure 1. CC3 impeller and vaned diffuser hardware. ${ }^{9}$ configurations, the choking flow rate.

The current research effort was undertaken in an attempt to investigate and understand the potential causes of the mis-prediction of centrifugal compressor impeller performance by RANS solvers. Figure 1 shows the impeller studied in this investigation, a centrifugal compressor known as CC3. This test compressor is a $4.54 \mathrm{~kg} / \mathrm{s}(10 \mathrm{lb} / \mathrm{m} / \mathrm{s})$ machine which was scaled up from a $1.658 \mathrm{~kg} / \mathrm{s}\left(3.655 \mathrm{lb}_{\mathrm{m}} / \mathrm{s}\right)$ Allison Engine design. The design exit corrected flow rate of the test compressor is $1.36 \mathrm{~kg} / \mathrm{s}(3.0 \mathrm{lb} / \mathrm{m} / \mathrm{s})$. The complete design description and hot shape geometry of the test compressor are given by McKain and Holbrook. ${ }^{6}$ The test compressor was used in aerodynamics studies at NASA Glenn Research Center. ${ }^{7-9}$ The impeller was tested in both vaned diffuser and vaneless diffuser configurations, and detailed measurements of the flow field were obtained. The data available for this test compressor qualifies it as a good candidate for further computational studies.

The CFD solver used in the current work is the TURBO Parallel Version 4 code. TURBO is a three-dimensional, viscous, unsteady RANS solver for turbomachinery flows. Flux vector splitting is used in evaluating flux Jacobians on the left hand side, while Roe's flux difference splitting is used to form a higher order TVD scheme for convective fluxes on the right hand side. The code can use multiple structured blocks with matching boundaries. Local time stepping can be employed to accelerate convergence for steady-state flows. Newton sub-iterations are applied to

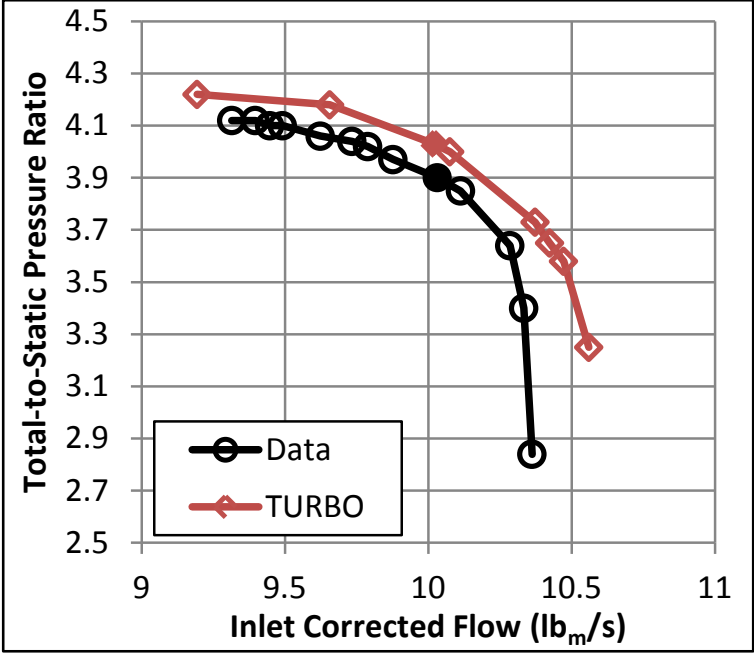

Figure 2. Computed and experimental total-tostatic pressure ratios of CC3 vaned diffuser configuration at design speed. unsteady flows to obtain a converged solution within each time step. ${ }^{10}$ Multiple blade rows can be modeled with sector periodic blade row meshes using a simple periodic boundary condition or with a single blade passage per blade row using phase lag boundary conditions. ${ }^{11,12}$ The default turbulence model in TURBO is the NASA/CMOTT $\mathrm{k}-\varepsilon$ turbulence model with wall functions. ${ }^{13}$ The code has previously been validated on various geometries. ${ }^{14,15}$

As a precursor to the current research effort, unsteady phase-lag simulations of the CC3 impeller with the vaned diffuser configuration were generated using TURBO. The resulting speed line at the design speed is shown in Fig. 2 alongside a comparison to the data. An over-prediction in choking flow rate was observed in the CFD result, and this speed line serves to illustrate a trend seen throughout the literature. ${ }^{2-5}$

The CFD results shown are converged, unsteady phaselag computations which were time-averaged over one revolution. Computed static pressures at the exit rating station were area-averaged. In these simulations, a grid of 
4.3 million points with resolution deemed to be typical of turbomachinery CFD was used. A row of three support struts upstream of the impeller, as well as leakage flows, were neglected in the computation. The computation overpredicted pressure ratio by $3.3 \%$ at the design point inlet corrected flow rate (shown as a filled symbol) and overpredicted choking flow rate by $1.9 \%$. The computation was run with ambient inlet pressure of 101,325 Pa, whereas the data was taken at approximately 86,000 $\mathrm{Pa}$. The computation was performed with the measured clearances, which are minimum tip clearances. If clearances are highly non-axisymmetric in the test article, modeling the minimum tip clearance may explain part of the over-prediction in the computed performance. The questions of clearance sensitivity and Reynolds number sensitivity are left for future work. Instead, it was hypothesized that the over-prediction of performance may be due to inaccurate simulation of the unsteady, non-uniform impeller discharge flow and the subsequent impact on associated time-mean aerodynamic blockage at the diffuser throat.

The scope of the research effort was narrowed to more closely examine the impeller exit flow. The focus of this paper is the CC3 impeller in its vaneless diffuser configuration. The compressor was modeled as a steady, periodic, single blade row computation in TURBO, which reduced computational expense and run-times. While the vaneless configuration obviates the impact of periodic unsteadiness, it was anticipated that lessons learned in modeling this simpler case may be useful in future work involving impellers and vaned diffuser configurations. The details of the compressor experiments are provided in the next section, followed by details of the computational effort.

\section{Details of the Experiment}

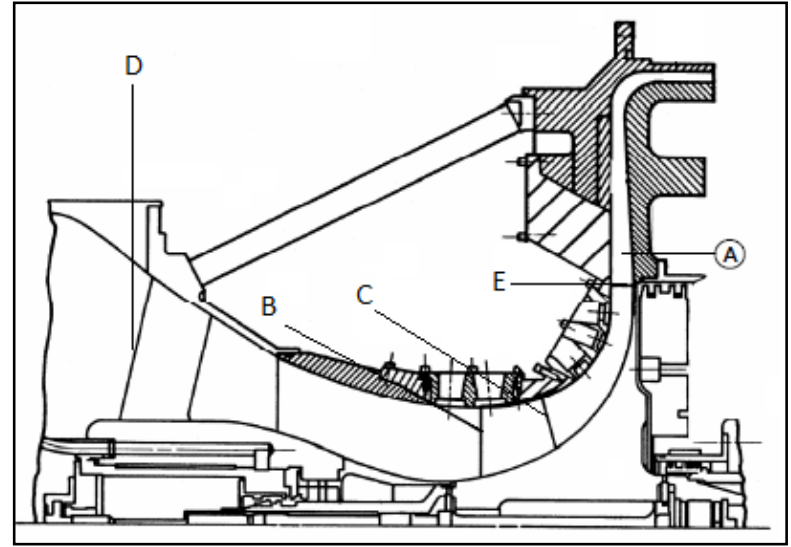

Figure 3. Cross-sectional drawing of the CC3 rig in the vaneless diffuser configuration. ${ }^{7} A$ is the exit rating station, $R / R_{T E}=1.18 ; B$ is the impeller main blade leading edge; $C$ is the impeller splitter blade leading edge; $D$ is the leading edge of a row of support struts; $E$ is the impeller trailing edge.

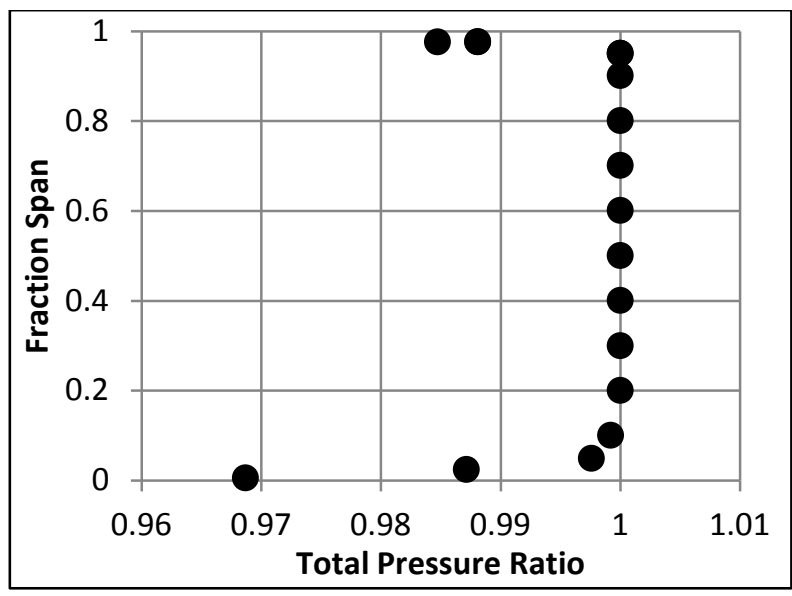

Figure 4. CC3 inlet survey data measured $2.54 \mathrm{~cm}$ (1.0 inch) upstream of impeller leading edge.
Table 1: CC3 impeller design parameters.

\begin{tabular}{ll}
\hline Impeller main blade count & 15 \\
Impeller splitter blade count & 15 \\
\hline Design corrected speed & $21,789 \mathrm{RPM}$ \\
Design inlet corrected flow rate & $4.54 \mathrm{~kg} / \mathrm{s}$ \\
Design exit corrected flow rate & $1.36 \mathrm{~kg} / \mathrm{s}$ \\
Impeller exit tip speed & $492 \mathrm{~m} / \mathrm{s}$ \\
Inlet blade height & $64 \mathrm{~mm}$ \\
Exit blade height & $17 \mathrm{~mm}$ \\
Leading edge tip radius & $105 \mathrm{~mm}$ \\
Trailing edge radius, $\mathrm{R}_{\mathrm{TE}}$ & $215.5 \mathrm{~mm}$ \\
Impeller backsweep & $50^{\circ}$ \\
\hline
\end{tabular}

The focus of the current work was the CC3 impeller in its vaneless diffuser configuration. A cross-section of the compressor rig is shown in Fig. 3. Table 1 summarizes important design parameters. The impeller consists of 15 main blades and 15 splitter blades having 50 degrees of backsweep from the radial direction. The design corrected speed was 21,789 RPM, and the measured minimum clearances at this speed were $0.1524 \mathrm{~mm}$ (0.006 inches) at the leading edge, 0.6096 $\mathrm{mm}$ (0.024 inches) at the midchord "knee" and 0.2032 $\mathrm{mm}$ (0.008 inches) at the trailing edge. ${ }^{7}$ At this clearance schedule and in the cold shape, the impeller hub at the trailing edge sat axially forward of the diffuser hub by $0.127 \pm 0.0508 \mathrm{~mm} \quad(0.005 \pm 0.002$ inches). This step in the hub was neglected in the computations.

Total pressure and total temperature exit rakes were located at eight evenly spaced circumferential locations at radius ratio $\mathrm{R} / \mathrm{R}_{\mathrm{TE}}=1.18$. Each total pressure rake had four elements located at $19.2 \%, 43.4 \%, 68.2 \%$, and $93.1 \%$ of span, and each total temperature rake had three elements located at $23.1 \%, 55.9 \%$, and $88.9 \%$ of span. Performance parameters were calculated using arithmetic averages of these rake elements. An inlet probe was used to acquire spanwise total pressure at a

American Institute of Aeronautics and Astronautics 
station $2.54 \mathrm{~cm}$ (1.0 inch) upstream of the impeller main blade leading edge (Fig. 4). Spanwise total pressure surveys were also acquired by using a constant-blockage probe immersed downstream of the impeller trailing edge at radius ratio $\mathrm{R} / \mathrm{R}_{\mathrm{TE}}=1.10$. Features which were neglected in the computations include a row of three inlet struts upstream of the impeller, impeller blade fillets, and backplate leakage paths. A set of 16 equally spaced $5 / 16^{\text {th }}$ inch diameter pins, which held together the diffuser hub and shroud, were located in the test compressor at radius ratio $\mathrm{R} / \mathrm{R}_{\mathrm{TE}}=1.45$. These structural elements were thought to be sufficiently far downstream of the exit rating station and were neglected in the computational model.

\section{Details of the Computations}

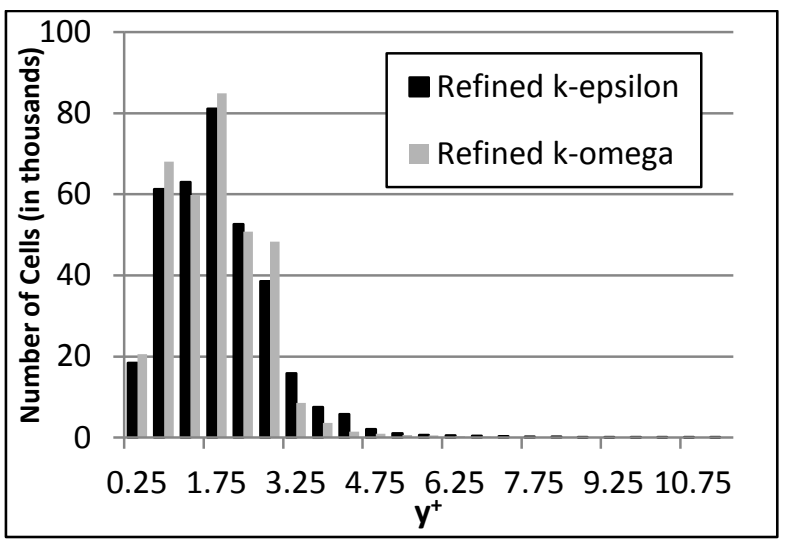

Figure 5. Histogram comparing $\mathrm{y}^{+}$of $\mathrm{k}-\varepsilon$ and $\mathrm{k}-\omega$ computations on the refined grid near design point.

The default turbulence model in TURBO Parallel Version 4 is the NASA/CMOTT $k-\varepsilon$ model with wall functions. ${ }^{13}$ In addition to the default $\mathrm{k}-\varepsilon$ model, Wilcox's k- $\omega$ turbulence model was used. ${ }^{16}$ The implementation of the $\mathrm{k}-\omega$ model does not include wall functions, and the $k-\omega$ model required a grid with sufficiently low $\mathrm{y}^{+}$so as to allow integration to the wall. Thus, two different grids were used in the current effort. A "baseline" grid with approximately 6.8 million points and $\mathrm{y}^{+}$on the order of 50 was used for $\mathrm{k}-\varepsilon$ computations with wall functions enabled. A "refined" grid containing approximately 14 million points was also generated, allowing integration to the wall for $k-\omega$ as well as $k-\varepsilon$ computations, in an effort to characterize the effect of varying grid resolution on aerodynamic performance. A histogram of $\mathrm{y}^{+}$for the $\mathrm{k}-\varepsilon$ and $\mathrm{k}-\omega$ computations on the refined grid is shown in Fig. 5. Typically, $\mathrm{y}^{+}$was less than 3 for a majority of the walls. Values of $\mathrm{y}^{+}$approached 5 along the blunt trailing edge of the impeller and at the shroud near the trailing edge of the suction side of the impeller, but these were within the viscous sublayer region. In all computational cases, inlet turbulence intensity was set to $1 \%$, and inlet turbulent eddy viscosity ratio was set to 100.0 . These values correspond to an inlet turbulence length scale of approximately $0.6 \%$ of the impeller inlet blade height.

TURBO solves the unsteady RANS equations on multiblock structured grids. All grids, boundary condition files, and block connectivity files for TURBO were generated using the Turbomachinery Gridding System (TGS). ${ }^{17}$ The measured tip clearances, which were minimum tip clearances, were included in the computational grid. Impeller blades and tip clearances were gridded with O-blocks, and H-blocks were used to connect the O-blocks around the impeller main and splitter blades. The block topology of the refined grid is shown in Fig. 6. The computational

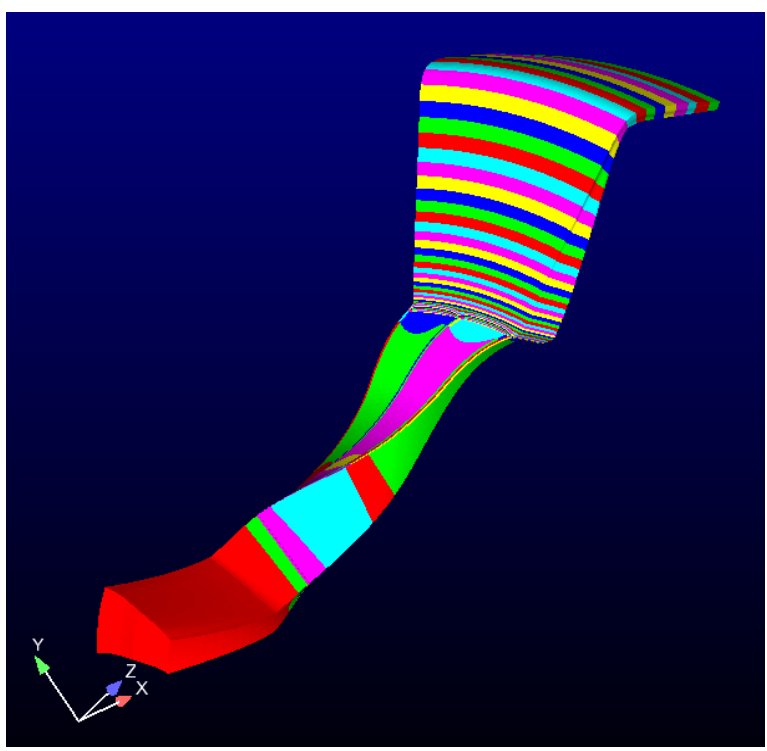

Figure 6. Block topology of the CC3 impeller and vaneless diffuser.

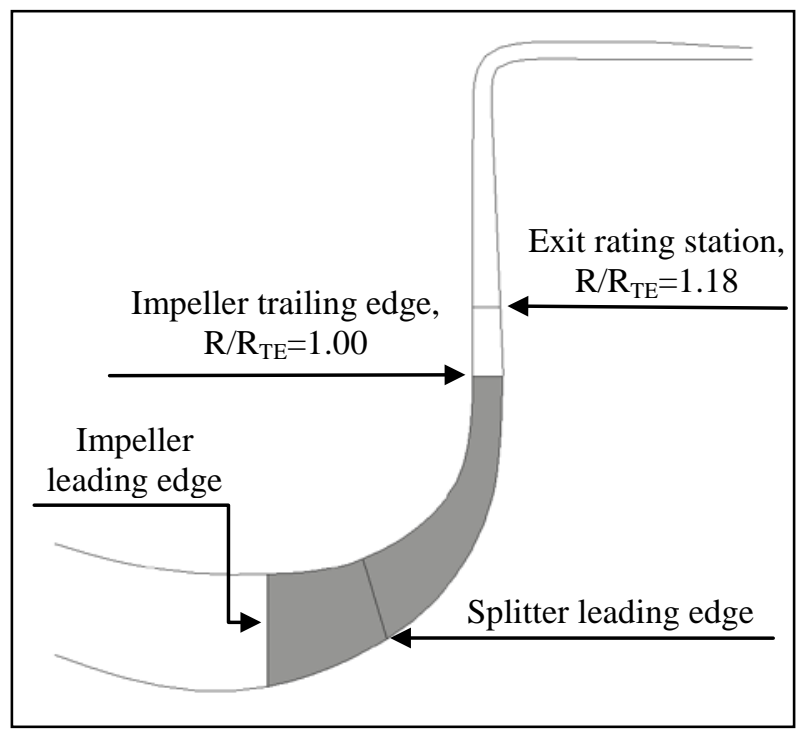

Figure 7. Meridional view of the CC3 impeller and vaneless diffuser model.

American Institute of Aeronautics and Astronautics 
domain consists of the axial inlet, an impeller and splitter passage, the vaneless diffuser, and the S-bend to the axial outlet. A meridional view of the computed flow path is shown in Fig. 7.

Steady, single passage periodic simulations were converged at design speed using local time stepping. Solid walls were modeled with no-slip boundary conditions and were assumed to be adiabatic. The specific heat coefficient ratio was assumed constant. The inlet boundary condition was specified with spanwise profiles of total pressure, total temperature, radial flow angle, and tangential flow angle. The total pressure profile, shown in Fig. 4, was the measured inlet probe survey data from the CC3 experiment. The computations were referenced to an inlet pressure of 101,325 $\mathrm{Pa}$, whereas the data was taken at reduced inlet pressures on the order of 85,500 $\mathrm{Pa}$. Inlet total temperature was assumed constant 288.15 Kelvin, and zero inlet tangential flow angle was assumed. The profile of inlet radial flow angle was found by linear interpolation between the geometry angles of the hub and shroud at the inlet. The computations were throttled from choke, up the speed line, but were not taken to the stall boundary. The operating point was set by using a radial equilibrium exit boundary condition, specifying exit static pressure at the shroud.

\section{Results}

Design speed computations of the impeller and vaneless diffuser were generated using TURBO. Three computed speed lines are compared to data in Fig. 8. The three computed results are the k- $\varepsilon$ result with wall functions on the baseline grid, and the $k-\varepsilon$ and $k-\omega$ results integrating to the wall on the refined grid. The computations were not taken to the stall boundary or deep into choke, as comparisons to the near-peak-efficiency data point were of interest in the current effort. The computed results were area-averaged in the circumferential direction at the exit rating station location, $\mathrm{R} / \mathrm{R}_{\mathrm{TE}}=1.18$. The area-averaging of the steady computations in the rotating frame of reference is analogous to the time-averaging done by the steady rake instrumentation, which is fixed in the stationary frame of reference while immersed in the unsteady rotor exit flow. The circumferential averaging yielded a spanwise profile of computed total pressure and total temperature. An arithmetic average of the values of total pressure and total temperature at those spanwise locations which correspond to the rake element locations was calculated, which was analogous to the experimental averaging approach.

The choking flow rates of the baseline and refined $k-\varepsilon$ computations agree to within $0.33 \%$ of each other. The $\mathrm{k}-$ $\omega$ computation choked at corrected flow $0.5 \%$ lower than the refined $k-\varepsilon$ computation. This difference is attributed to a slightly larger growth in the boundary layer in the k- $\omega$ result along the pressure side of the main impeller blade. The larger boundary layer results in increased aerodynamic blockage, reducing both effective flow area and choking flow rate. The $\mathrm{k}-\omega$ computation under-predicts choking flow rate by $1.22 \%$ as compared to data.

Both $k-\varepsilon$ results under-predict total pressure ratio and efficiency as compared to the data. The k- $\omega$ computations better match the data on these performance parameters. The near-peak-efficiency data point and the computed operating points at nearby inlet corrected flow rates are shown as filled symbols in Fig. 8. These operating points were compared. All three sets of computations over-predicted the work done by the impeller, as shown by the total temperature curves which are approximately $0.6 \%$ higher than the data. Compared to the near-peak-efficiency data point, the baseline k- $\varepsilon$ result was 7.2 points lower in efficiency and $7 \%$ lower in total pressure ratio. The refined k- $\varepsilon$ result was 8.3 points lower in efficiency and $8 \%$ lower in total pressure ratio than the data. The k- $\omega$ result was 1.2 points lower in efficiency and $0.6 \%$ higher in total pressure ratio than the data. In this case, the choice of turbulence model evidently had a much greater impact on the flow field than the selected level of grid density.

A comparison of shroud static pressures at these operating points is shown in Fig. 9. The data have been normalized by the plenum pressure of 85,360 $\mathrm{Pa}$ and the computations are referenced to inlet total pressure 101,325 $\mathrm{Pa}$. The shroud static pressures of the data, the $\mathrm{k}-\omega$ case, and both $\mathrm{k}-\varepsilon$ cases show very good agreement from the inlet to the impeller trailing edge. Downstream of the trailing edge, the baseline and refined k- $\varepsilon$ computations have virtually identical shroud static pressures. The $k-\varepsilon$ computations have reduced pressure recovery through the diffuser as compared to the data and to the $\mathrm{k}-\omega$ computation. In the vaneless diffuser, the $\mathrm{k}-\omega$ simulation matches the data well through the initial half of the diffuser, but over-predicts pressure recovery towards the S-bend and beyond.

In Fig. 10 spanwise profiles of the computed flow angles (from tangential) and total pressures are compared against the profiles measured by the constant-blockage probe at radius ratio $R / R_{T E}=1.10$. This is a location nearly midway between the impeller trailing edge and the exit rating station, where the shroud static pressures of the k- $\omega$ case and the k- $\varepsilon$ cases are diverging. The uncertainty of the measured flow angle is not obvious, so measured flow angles are shown with both $\pm 1^{\circ}$ and $\pm 3^{\circ}$ error bars for reference. The comparison shows that the baseline and refined $\mathrm{k}-\varepsilon$ computations both yield nearly identical total pressure and tangential flow angle profiles. The flow angle profiles indicate that the $\mathrm{k}-\varepsilon$ computations have a region of reversed flow (i.e. negative tangential flow angle) in the top $25 \%$ of the passage. This large region of reversed flow is an aerodynamic blockage which causes higher velocities and 


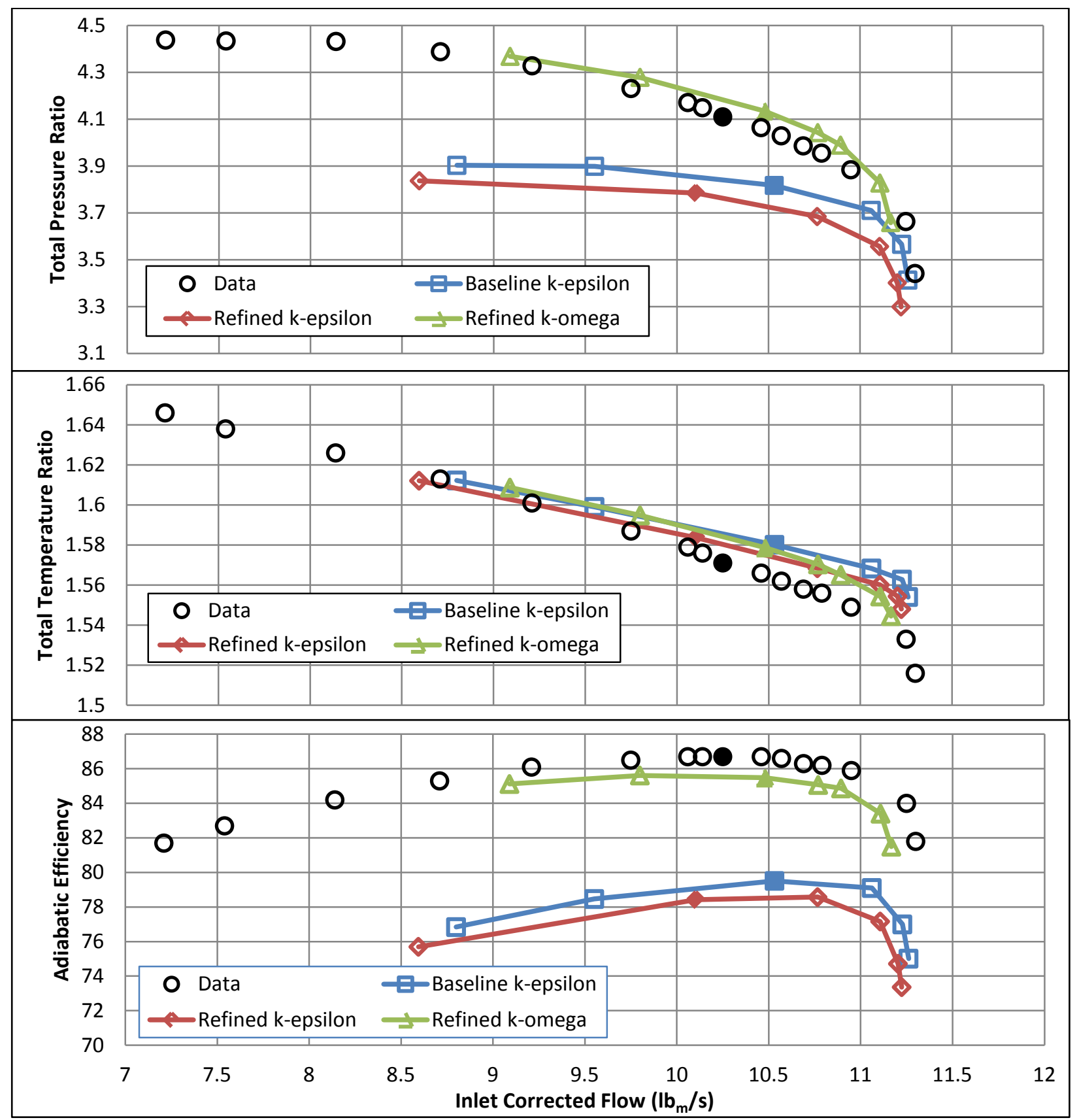

Figure 8. Total pressure ratio, total temperature ratio, and adiabatic efficiency of CC3 vaneless diffuser configuration at design speed. Note: the computed speed lines were not run to stall.

thus higher flow angles in the bottom $75 \%$ of the passage, as compared to the data. The reversed flow near the shroud is evidently the cause of the large deficit in total pressure observed in the $\mathrm{k}-\varepsilon$ computations, which disagree with the measured pressure profile above 55\% span. The data indicates reversed flow within the top 10-15\% of span near the shroud. The flow angle profile from the $k-\omega$ computation agrees with the data in this regard. The k- $\omega$ computation over-predicts flow angle in the bottom $50 \%$ of span, but to less of a degree than either $\mathrm{k}-\varepsilon$ computation. The k- $\omega$ computation misses the pressure deficit seen in the survey data above $75 \%$ span.

In Fig. 11, a comparison of flow angles is made between the three computations at two locations; just downstream of the trailing edge $\left(\mathrm{R} / \mathrm{R}_{\mathrm{TE}}=1.01\right)$, where the shroud static pressures are in agreement, and at the exit rating station $\left(R / R_{T E}=1.18\right)$, where there is a large difference in shroud static pressures. The flow angle profiles of the three computations match well near the trailing edge. At $\mathrm{R} / \mathrm{R}_{\mathrm{TE}}=1.01$, the flow angle profiles of the $\mathrm{k}-\varepsilon$ cases 


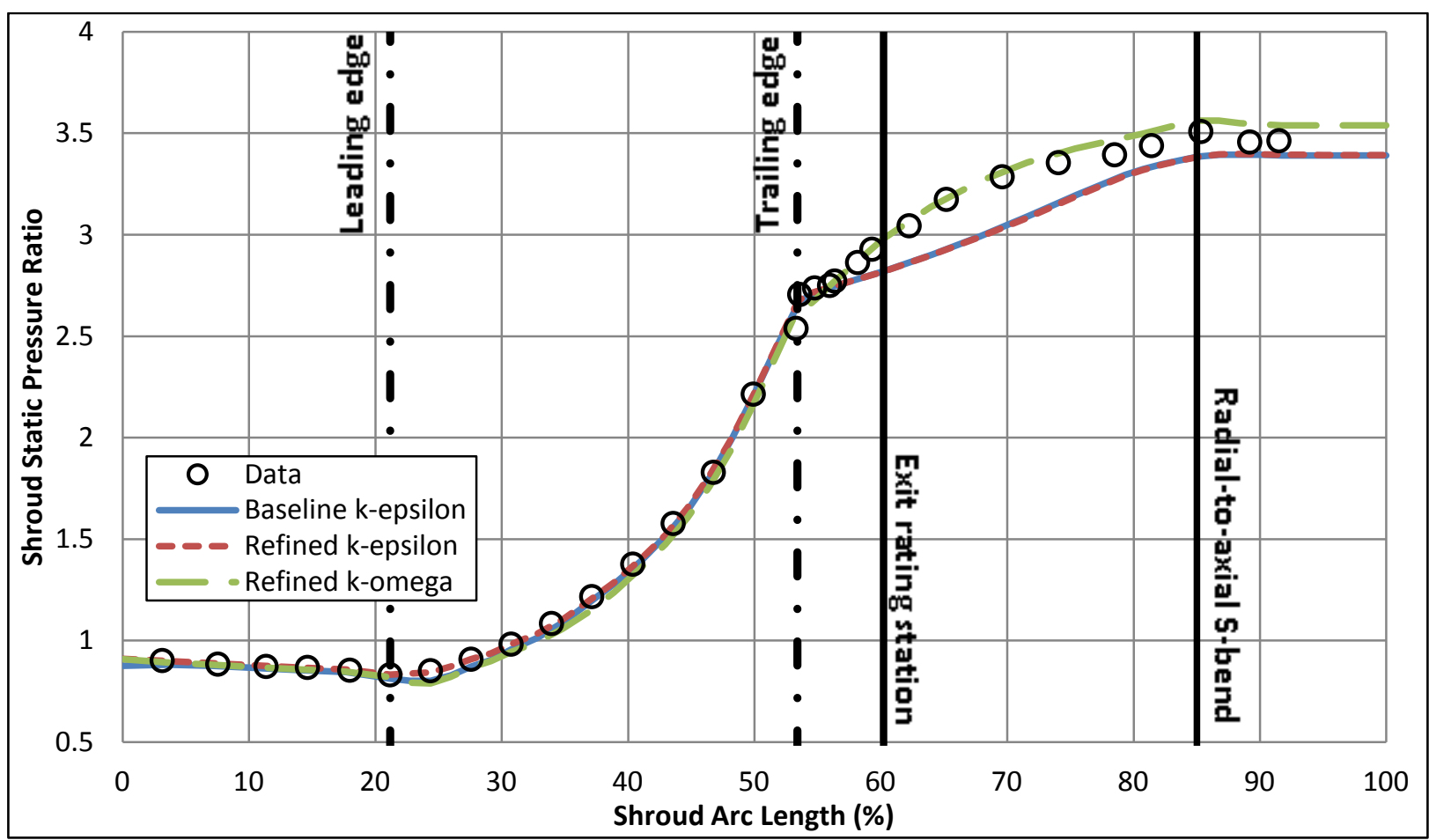

Figure 9. Computed and experimental static pressure ratio along the shroud surface. Vertical lines mark geometry features of interest.

match each other to within $\pm 0.5^{\circ}$, and the $\mathrm{k}-\omega$ case matches the $\mathrm{k}-\varepsilon$ cases within $\pm 1.5^{\circ}$, with slight disagreement above $95 \%$ span. The $\mathrm{k}-\varepsilon$ cases indicate reversed flow beginning at $97 \%$ span, the $\mathrm{k}-\omega$ case indicates reversed flow at $95 \%$ span. There is large disparity between the $k-\varepsilon$ cases and the $k-\omega$ case at radius ratio $R / R_{T E}=1.18$. The flow angle profiles of the two k- $\varepsilon$ computations, which agree to within $\pm 1.0^{\circ}$, indicate that the flow has reversed in nearly $30 \%$ of the span near the shroud. The $\mathrm{k}-\omega$ case has reversed flow in only the upper $5 \%$ of span near the shroud.

The deficit in static pressure recovery seen in the k- $\varepsilon$ computations is attributed to the large region of reversed flow. This region is shown in Fig. 12, which plots the computed radial velocity contours near the impeller trailing edge, $\mathrm{R} / \mathrm{R}_{\mathrm{TE}}=1.01$, and at the exit rating station, $\mathrm{R} / \mathrm{R}_{\mathrm{TE}}=1.18$. The contours near the trailing edge show the splitter blade in the middle of the plot, and the main blade at the left and right sides of the plot. At $R / R_{T E}=1.01$, the low-
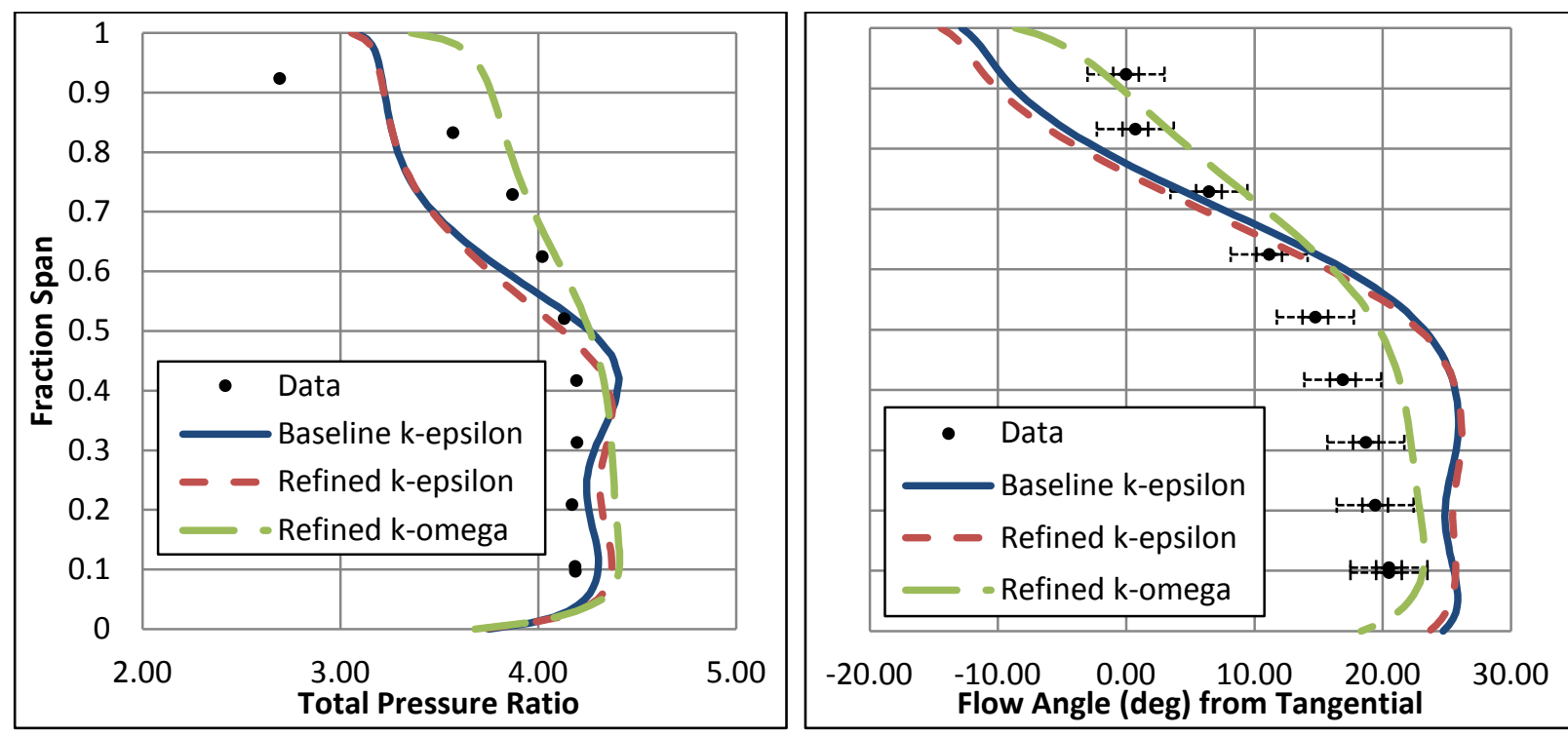

Figure 10. Area-averaged computed total pressure ratio (left) and flow angle (right) compared to survey data at radius ratio $R / R_{T E}=1.10$. 

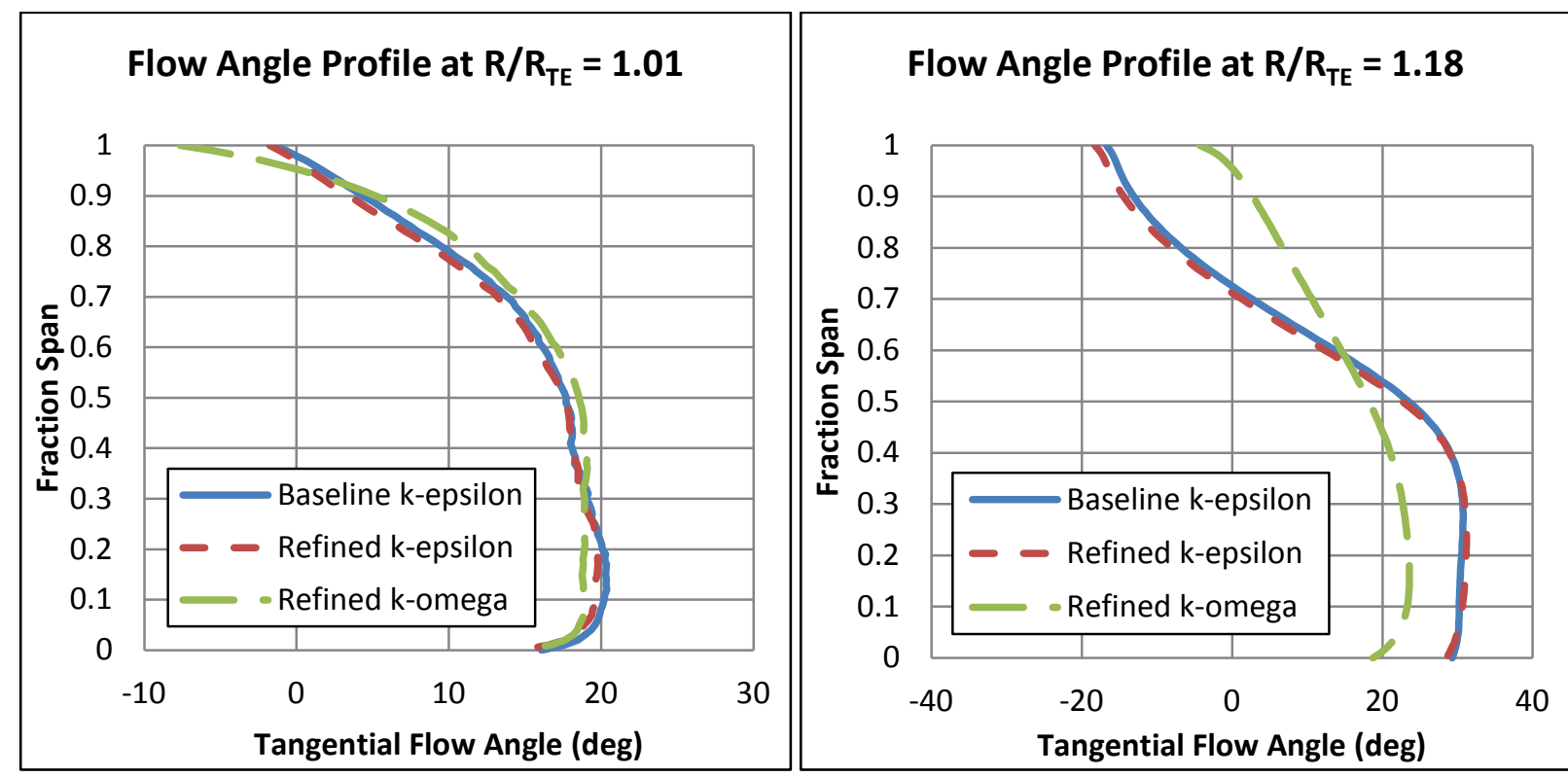

Figure 11. Area-averaged flow angles comparing results of the $k-\varepsilon$ and $k-\omega$ turbulence models at radius ratios $R / R_{T E}=1.01$ (left) and $R / R_{T E}=1.18$ (right).

momentum regions near the hub that extend into mid-span are associated with the impeller blade wakes. The low radial velocity region at the shroud is the tip clearance flow. The spanwise extent of the clearance flow is slightly larger in the $k-\varepsilon$ computations as compared to the $k-\omega$ computation, indicating a stronger tip clearance vortex in the $\mathrm{k}-\varepsilon$ computations. The flow fields of the two different turbulence models are qualitatively and quantitatively similar at the trailing edge. If performance is computed from the inlet plane to the plane defined by $\mathrm{R} / \mathrm{R}_{\mathrm{TE}}=1.01$, the refined

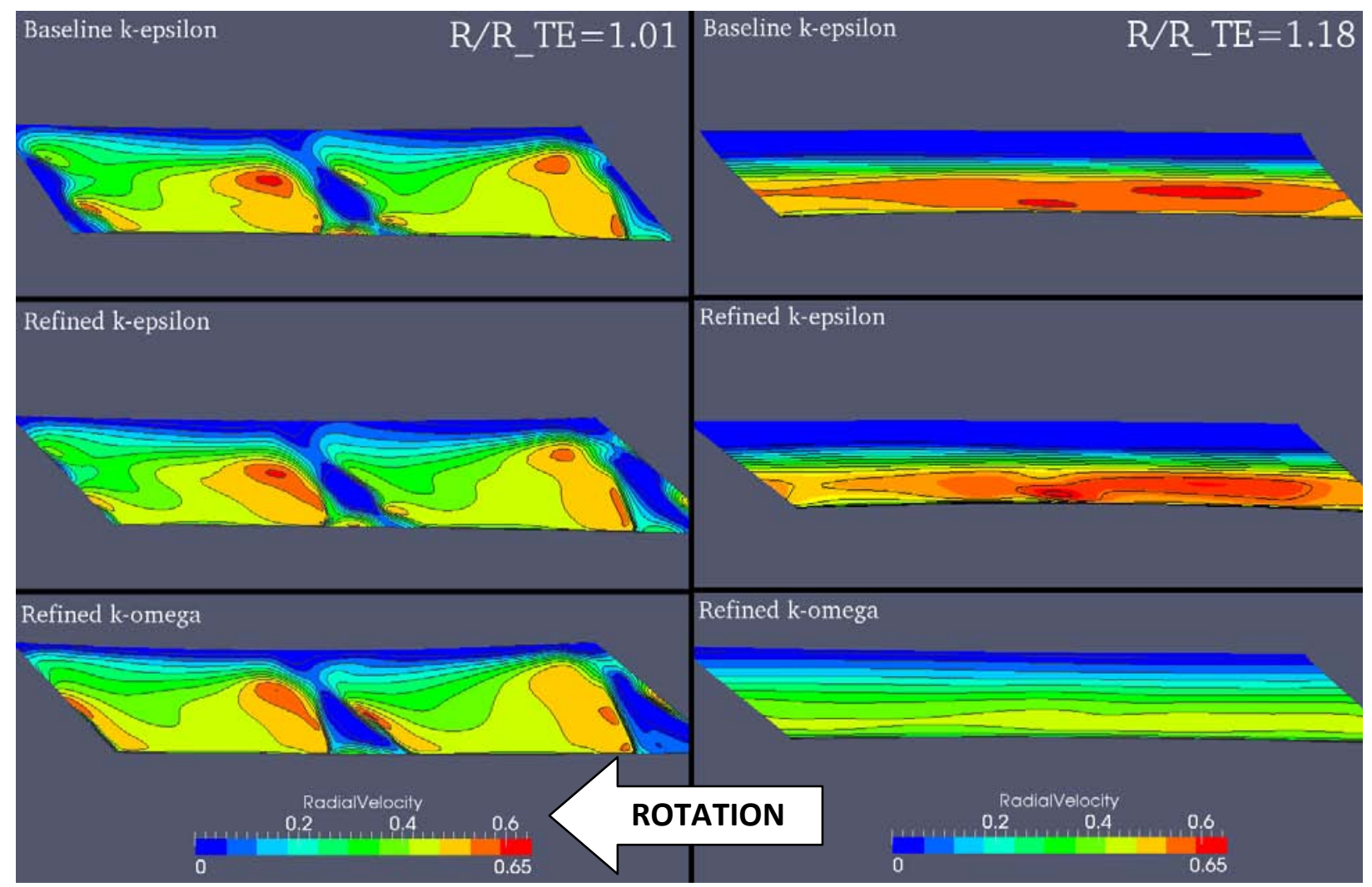

Figure 12. Radial velocity contours (normalized by $287.6 \mathrm{~m} / \mathrm{s}$ ) at the trailing edge of the impeller, $R / R_{\mathrm{TE}}=1.01$ (left), and at the exit rating station $R / R_{T E}=1.18$ (right). Impeller blade rotation is right-to-left. 
$\mathrm{k}-\varepsilon$ and $\mathrm{k}-\omega$ computations agree to within $0.7 \%$ in total pressure ratio and to within 1.0 point in adiabatic efficiency. At the exit rating station $\mathrm{R} / \mathrm{R}_{\mathrm{TE}}=1.18$, the two $\mathrm{k}-\varepsilon$ computations are qualitatively very different from the $\mathrm{k}-\omega$ computation, and the computed performance parameters at $\mathrm{R} / \mathrm{R}_{\mathrm{TE}}=1.18$ amount to a $8.4 \%$ difference in total pressure ratio and 7.0 point difference in adiabatic efficiency between the refined $k-\varepsilon$ and $k-\omega$ computations.

Figure 13 plots entropy on planes of constant radius through the last $30 \%$ of impeller chord to show loss development through the passage. In the figure, the pressure side of the main blade is on the right, the splitter blade is in the middle, and the suction side of the main blade is on the left. The computations differ at the corner formed

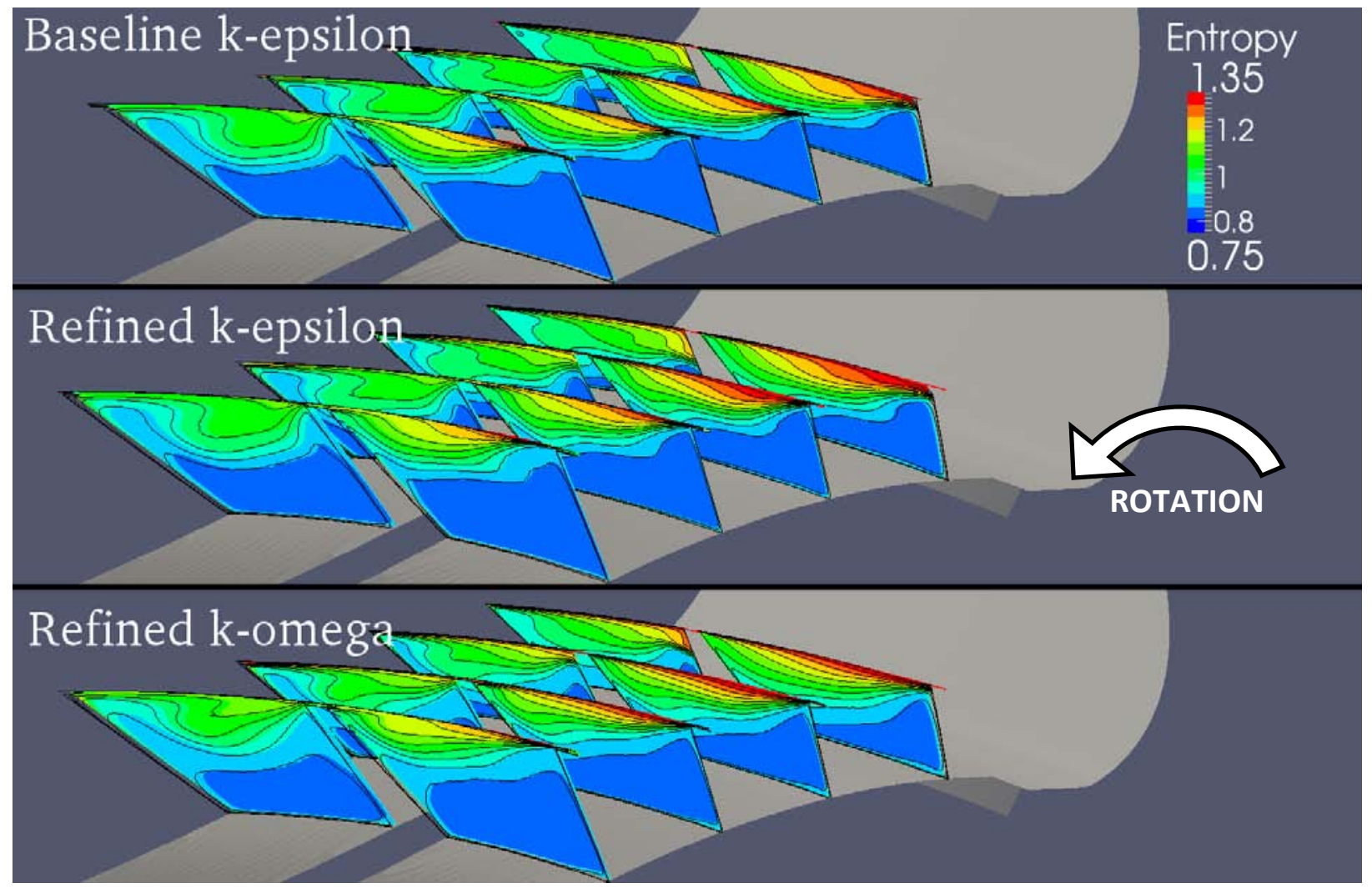

Figure 13. Entropy contours at constant-radius planes over the last $30 \%$ of impeller chord, with the splitter blade shown in the center of the passage.

by the pressure side of the main blade and the shroud. The higher magnitude of entropy in the refined $k-\varepsilon$ case indicates larger loss due to a stronger tip clearance flow than in the baseline $\mathrm{k}-\varepsilon$ case. The spanwise extent of the high entropy region is smaller in the $k-\omega$ case, indicating a weaker tip clearance flow than in the $k-\varepsilon$ cases. Qualitatively, the entropy contours among the three computations are similar through the trailing edge.

Figure 14 plots radial velocity through the radial diffuser and axial velocity after the Sbend. These velocities were area-averaged in the circumferential direction for the $k-\varepsilon$ and $k-\omega$ results on the refined grid. This shows the extent of the reversed flow through the diffuser along the shroud in each case. In both computations, the impeller exit flow is similar, but differences arise in the diffuser. Both computations show reversed flow at the shroud

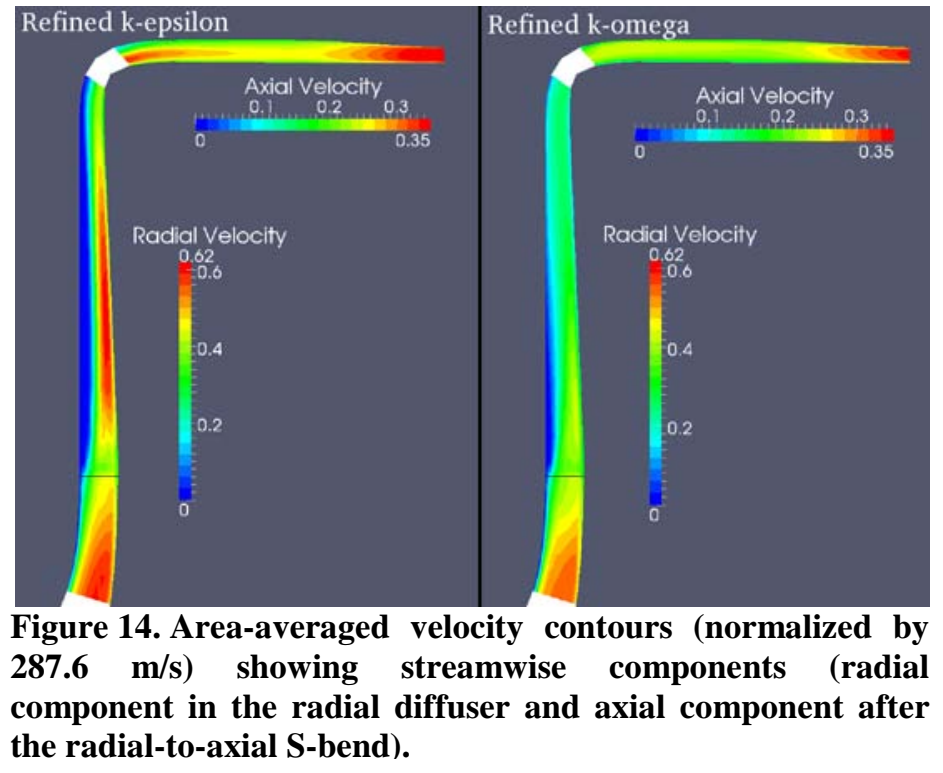


at close proximity to the impeller trailing edge. In the k- $\varepsilon$ computation, the reversed flow persists up the radius of the diffuser along the shroud surface. In the $\mathrm{k}-\omega$ case, there is a relatively small bubble of reversed flow which weakens as radius increases through the diffuser. The separated region in the $\mathrm{k}-\varepsilon$ cases reattaches through the S-bend and there is no negative axial velocity at the end of the computational domain. The diffuser flow field had large sensitivity upon the choice of turbulence model. A discussion on the differences in the diffuser flow field is presented next, as well as comments and suggestions for future work.

\section{Discussion}

A better understanding of the cause of the disparate diffuser flow fields between the k- $\varepsilon$ and $k-\omega$ computations is of interest for future work. The flow fields indicate that the $k-\omega$ computation has greater turbulent mixing in the diffuser, which causes the shroud separation to weaken and mix out in a manner that is not occurring in the k- $\varepsilon$ computations. Figure 15 shows a band of high turbulence kinetic energy (TKE) in the diffuser of the k- $\varepsilon$ case which is not evident in the $k-\omega$ case. This region is attributed to the shear layer at approximately $70 \%$ span due to the interface of the separated flow and core flow in the k- $\varepsilon$ case. Generally, it was observed in the k- $\omega$ computation that the levels of both production and dissipation of TKE were higher than the k- $\varepsilon$ computations near the shroud within the impeller passage, but these terms were lower than the k- $\varepsilon$ computations through the diffuser.

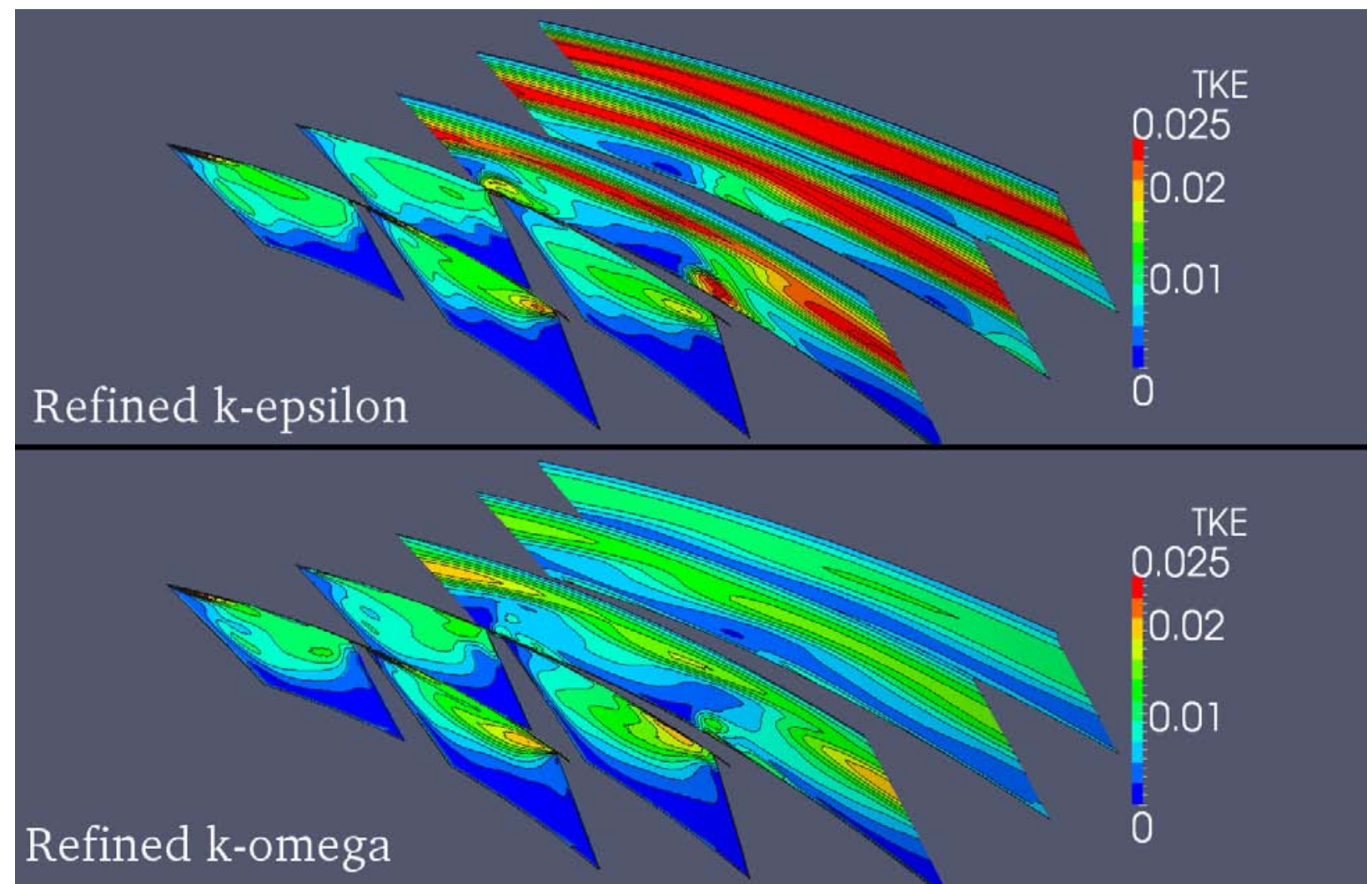

Figure 15. Turbulence kinetic energy at constant-radius planes from $R / R_{T E}=0.9$ to $R / R_{T E}=1.18$. The splitter blade is shown in the center of the passage.

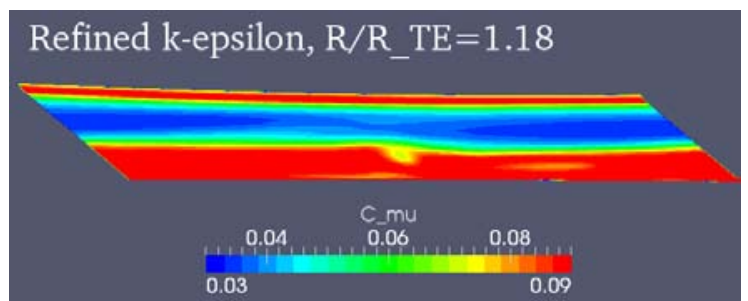

Figure 16. $C_{\mu}$ of the refined grid $k-\varepsilon$ computation at radius ratio $R / R_{T E}=1.18$ (exit rating station).
It may be of interest in future work to generate a simulation using a standard k- $\varepsilon$ model, which sets constant $\mathrm{C}_{\mu}=0.09$ in the turbulent eddy viscosity equation rather than a computed value as in the CMOTT k- $\varepsilon$ model. As shown in Fig. 16, the computed $C_{\mu}$ in the refined CMOTT k- $\varepsilon$ result yields values lower than 0.09 in the shear layer region at $70 \%$ span in the diffuser at the exit rating station. A standard $\mathrm{k}-\varepsilon$ model would increase $\mathrm{C}_{\mu}$ in this region, resulting in increased turbulent eddy viscosity and turbulent mixing, which may mix out or weaken the separation observed in the 
current k- $\varepsilon$ cases.

The current results suggest that the flow developing through the radial diffuser may largely depend on the selection of turbulence model. In a vaned diffuser configuration, a strong separation entering the diffuser impacts the aerodynamic blockage, which affects the pressure rise and flow capacity in the diffuser. The impact of different turbulence models on this separation, and thus on the stage performance, should be analyzed in future work. The vaned diffuser computation poses a more complicated problem, as the blockage will also be dependent upon the unsteadiness due to the impeller/vane interaction in a vaned diffuser configuration.

\section{Conclusion}

A computational study of the CC3 impeller in its vaneless diffuser configuration was undertaken. TURBO CFD simulations were generated on: 1) a baseline grid using the default CMOTT k- $\varepsilon$ turbulence model, 2) a refined grid using the default CMOTT k- $\varepsilon$ turbulence model, and 3) a refined grid using Wilcox's k- $\omega$ turbulence model. The results indicated that all three computational cases matched the data well from the inlet, through the impeller, and up to the trailing edge. The area-averaged flow angle profiles of the baseline and refined k- $\varepsilon$ cases matched to within $\pm 0.5^{\circ}$ just downstream of the impeller trailing edge $\left(\mathrm{R} / \mathrm{R}_{\mathrm{TE}}=1.01\right)$. At this radius ratio, the flow angle profile of the $\mathrm{k}-\omega$ case matched both $\mathrm{k}-\varepsilon$ cases to within $\pm 1.5^{\circ}$, and the refined $\mathrm{k}-\varepsilon$ and $\mathrm{k}-\omega$ computations agreed to within $0.7 \%$ in total pressure ratio and to within 1.0 point in adiabatic efficiency. Beyond the trailing edge and into the diffuser, the $\mathrm{k}-\omega$ computation continued to match the data, but the k- $\varepsilon$ computations yielded pressure and efficiency deficits as compared to data due to a large reversed flow region extending from the shroud surface of the vaneless diffuser. At the experimental exit rating station $\left(\mathrm{R} / \mathrm{R}_{\mathrm{TE}}=1.18\right)$, the baseline $\mathrm{k}-\varepsilon$ computation is 7.2 points lower in efficiency and $7 \%$ lower in total pressure ratio than the data. The refined $k-\varepsilon$ computation is 8.3 points lower in efficiency and $8 \%$ lower in total pressure ratio than the data. The $\mathrm{k}-\omega$ computation is 1.2 points lower in efficiency and $0.6 \%$ higher in total pressure ratio than the data. It was shown that the potential impact of the choice of turbulence model may be quite large on the diffuser flow field.

\section{Acknowledgments}

This effort is sponsored by the Rotary Wing Project of the NASA Fundamental Aeronautics Program. The authors acknowledge and thank Dr. Vikram Shyam for providing source code for the k- $\omega$ turbulence model implementation in TURBO and for helpful discussions. The authors would like to thank Dr. Gerard E. Welch of NASA Glenn Research Center and Dr. Ali Ameri of The Ohio State University for technical review of this paper and for insightful discussions. The authors also thank Mr. Edward P. Braunscheidel of NASA Glenn Research Center, and Dr. Wai-Ming To and Mr. Richard A. Mulac of the University of Toledo for numerous helpful technical discussions.

\section{References}

${ }^{1}$ Welch, G. E., Hathaway, M. D., Skoch, G. J., Snyder, C. A., "Rotary-wing relevant compressor aero research and technology development activities at Glenn Research Center,” NASA TM-217280, 2012.

${ }^{2}$ Chen, J. P., Webster, R. S., Hathaway, M. D., Herrick, G. P., and Skoch, G. J., "Numerical Simulation of Stall and Stall Control in Axial and Radial Compressors,” AIAA Paper No. AIAA-2006-418, 2006.

${ }^{3}$ Lurie, E. A., Van Slooten, P. R., Medic, G., Mulugeta, M. M., Holley, B. M., Feng, J., Sharma, O., and Ni, R., "Design of a High Efficiency Compact Centrifugal Compressor for Rotorcraft Applications", American Helicopter Society 67" Forum, 2011.

${ }^{4}$ Smythe, C. J., “Forced Response Predictions in Modern Centrifugal Compressor Design,” M.S. Thesis, Department of Aeronautics and Astronautics, Massachusetts Institute of Technology, 2005.

${ }^{5}$ Tarr, D. L., "Scaling of Impeller Response to Impeller-Diffuser Interactions in Centrifugal Compressors,” M.S. Thesis, Department of Aeronautics and Astronautics, Massachusetts Institute of Technology, 2008.

${ }^{6}$ McKain, T. F., and Holbrook, G. J., "Coordinates for a High Performance 4:1 Pressure Ratio Centrifugal Compressor," NASA CR-204134, 1997.

${ }^{7}$ Skoch, G. J., Prahst, P. S., Wernet, M. P., Wood, J. R., and Strazisar, A. J., "Laser Anemometer Measurements of the Flow Field in a 4:1 Pressure Ratio Centrifugal Impeller,” ASME Turbo Expo, Paper No. 97-GT-342, 1997.

${ }^{8}$ Wernet, M. P., Bright, M. M., and Skoch, G. J., “An Investigation of Surge in a High-Speed Centrifugal Compressor Using Digital PIV,” J. Turbomachinery, Vol. 123, Apr. 2001, pp. 418-218.

${ }^{9}$ Larosiliere, L. M., Skoch, G. J., and Prahst, P. S., “Aerodynamic Synthesis of a Centrifugal Impeller Using Computational Fluid Dynamics and Measurement,” J. Propulsion and Power, Volume 15, Number 5, 1999, pp. 623-632.

${ }^{10}$ Chen, J. P., and Whitfield, D. L., "Navier-Stokes Calculations for the Unsteady Flowfield of Turbomachinery,” AIAA Paper No. AIAA-93-0676, 1993.

${ }^{11}$ Chen, J. P., and Barter, J., "Comparison of Time-Accurate Calculations for the Unsteady Interaction in Turbomachinery Stages,” AIAA Paper No. AIAA-98-3293, 1998. 
${ }^{12}$ Chen, J. P., and Briley, W. R., “A Parallel Flow Solver for Unsteady Multiple Blade Row Turbomachinery Simulations,” ASME Turbo Expo, Paper No. 2001-GT-0348, 2001.

${ }^{13}$ Zhu, J., and Shih, T. H., "CMOTT Turbulence Module for NPARC,” NASA CR-204143, 1997.

${ }^{14}$ Turner, M. G., “Multistage Turbine Simulations with Vortex-Blade Interaction,” Journal of Turbomachinery, Vol. 118, Oct. 1996, pp. 643-653.

${ }^{15}$ Van Zante, D. E., To, W., and Chen, J. P., "Blade Row Interaction Effects on the Performance of a Moderately Loaded NASA Transonic Compressor Stage,” ASME Paper GT-2002-30575, 2002.

${ }^{16}$ Shyam, V., Ameri, A., and Chen, J. P., "Validation of Heat Transfer and Film Cooling Capabilities of the 3-D RANS Code TURBO,” NASA TM-216738, 2010.

${ }^{17}$ Beach, T. A., “Centrifugal Compressor Analysis using the TURBO Flow Solver,” NASA CR to be published, 2013. 